\title{
Spezielle Beatmungssituationen in der Neonatologie
}

Ulrich $\mathrm{H}$. Thome

\section{Übersicht}

Einleitung

Hochfrequenzbeatmung

Beatmung beim pulmonalen

interstitiellen Emphysem

Zusammenfassung

\section{Einleitung}

In diesem Beitrag sollen 2 Sonderfälle der mechanischen Beatmung in Ergänzung eines vorangegangenen Artikels aus Ausgabe 4/15 dieser Zeitschrift [1] behandelt werden: zum einen die Funktionsweise, Indikationen und Ergebnisse der Hochfrequenzbeatmung und zum anderen das Vorgehen beim Auftreten eines pulmonalen interstitiellen Emphysems.

\section{Hochfrequenzbeatmung}

Die Hochfrequenzbeatmung (High Frequency Ventilation [HFV] oder High Frequency Oscillation Ventilation [HFOV]) wurde in Münster von P. P. Lunkenheimer [2] entwickelt. Später stieß auch eine Arbeitsgruppe in Kanada durch Zufall auf die Möglichkeit, mit hochfrequenten Oszillationen den Gasaustausch aufrechtzuerhalten [3]. Die Hochfrequenzbeatmung ermöglicht bei erheblicher Steigerung der Beatmungsfrequenz auf 7 - 15 Hertz (420 - 900 Atemzüge/Minute) eine erhebliche Verringerung der Tidalvolumina. Außerdem sind Inspiration und Exspiration bei diesen hohen Frequenzen immer inkomplett, was sich in einer in den Atemwegen gedämpften Druckkurve widerspiegelt und das alveoläre Tidalvolumen weiter reduziert. Die Spitzenund Taldrücke erreichen nicht mehr die Alveolen, in denen nur noch geringe Druckamplituden messbar sind (Abb. 1). Die Gasfüllung der Alveolen entspricht

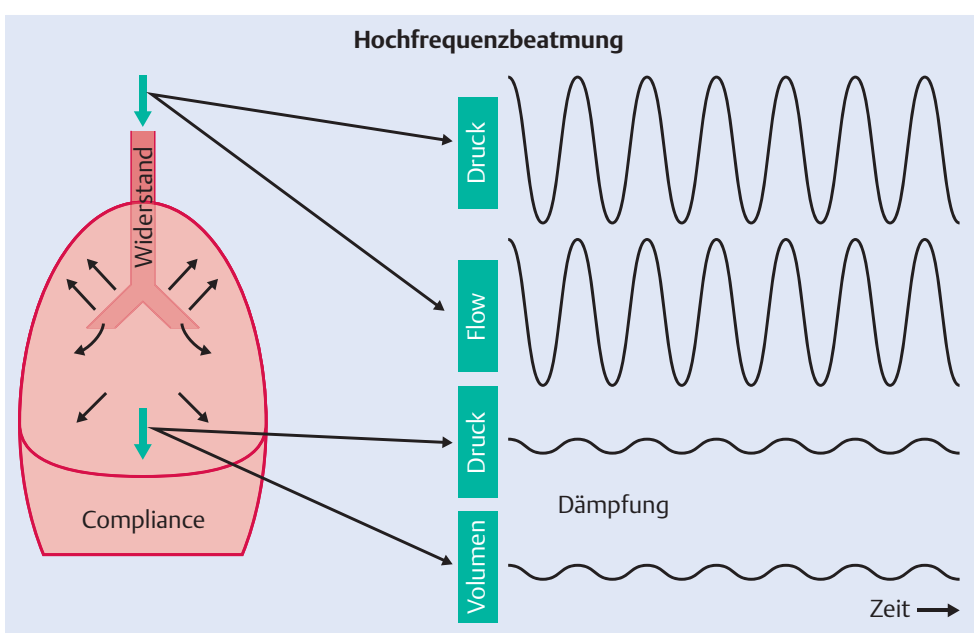

Abb. 1 Druck- und Flow-Kurven an verschiedenen Stellen der Atemwege während der Hochfrequenzbeatmung. Die Dämpfung bewirkt immer kleinere Amplituden mit zunehmender Entfernung vom Tubuseingang.

somit weitgehend dem mittleren Atemwegsdruck. Inspiratorische Überdehnung und exspiratorische Atelektasen werden vermieden.

Aufgrund der inkompletten In- und Exspiration und der Dämpfung der Druckkurve spielt die normale Konvektion für den Gastransport in den Atemwegen nur eine untergeordnete Rolle. Es kommt durch Turbulenzen in den großen Atemwegen und radiales Mischen in den kleinen Atemwegen zu einer intensiven Durchmischung des Atemgases bis hinein in die Alveolen, 


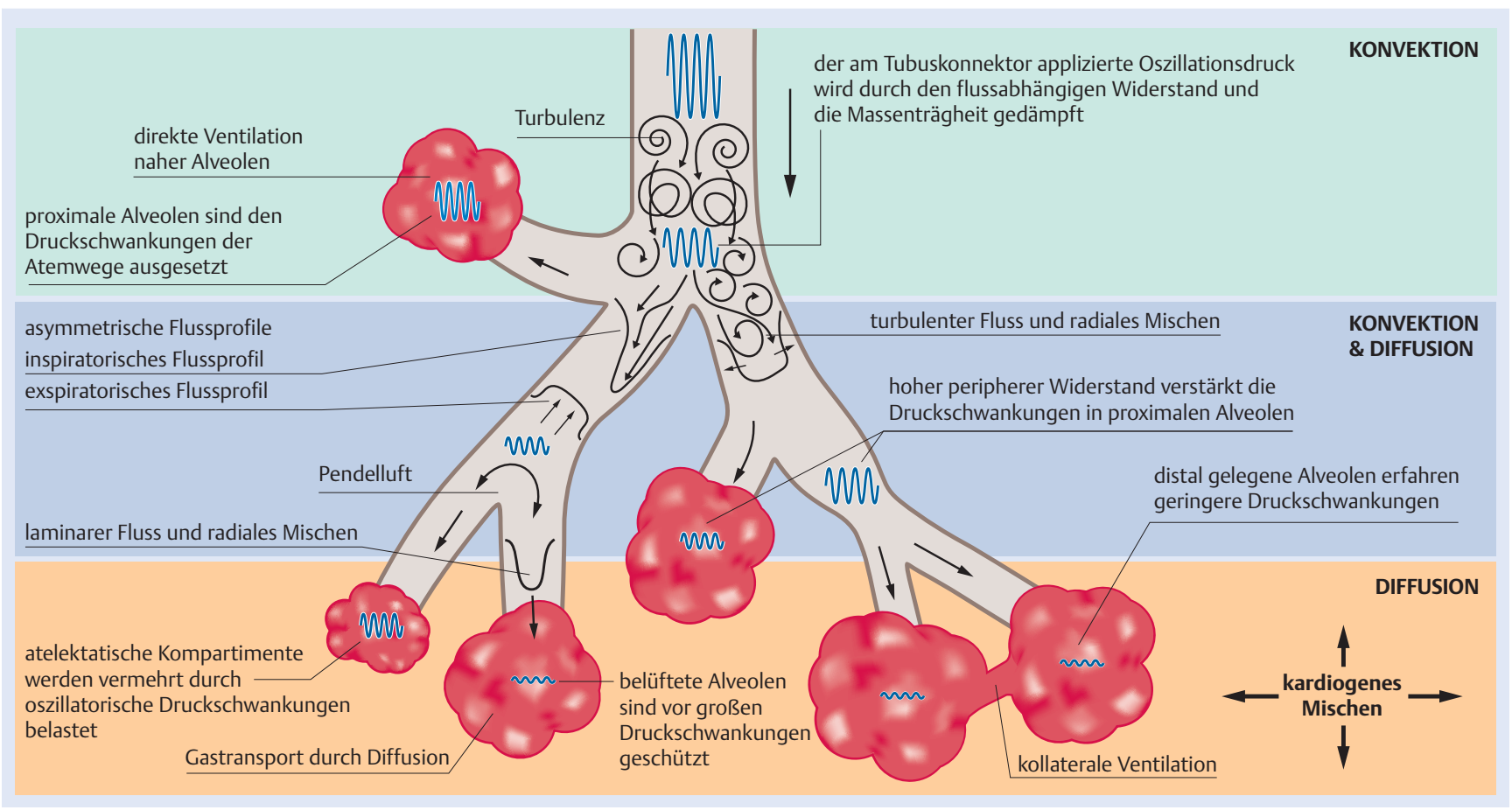

Abb.2 Mechanismen des Gastransports unter Hochfrequenzbeatmung. Der Gasaustausch funktioniert dadurch auch mit Tidalvolumina, die kleiner sind als der Totraum [4].

Abb. 3 Flussdiagramm zur initialen Einstellung und weiteren Steuerung des mittleren Atemwegsdrucks (MAP) [5].

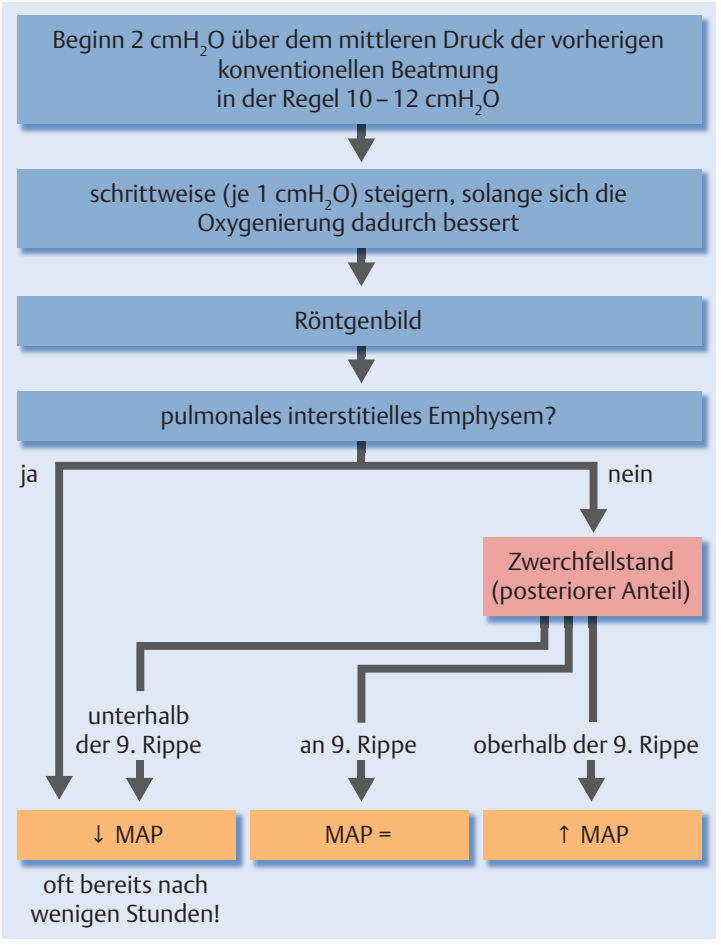

sodass entlang der Konzentrationsgradienten Sauerstoff in die Lunge hinein und $\mathrm{CO}_{2}$ aus der Lunge herausbefördert wird (Abb.2).
Für die Einstellung der Hochfrequenzbeatmung müssen folgende Parameter gewählt werden:

- mittlerer Atemwegsdruck

- Oszillationsamplitude

- Oszillationsfrequenz

- Inspirations- zu Exspirationsverhältnis (I:E-Verhältnis)

Bei manchen Geräten muss auch der Bias-Flow, d.h. die Durchströmung des Beatmungsschlauchsystems, eingestellt werden, andere regeln ihn automatisch. Bei manueller Regelung sollten die Empfehlungen des Herstellers beachtet werden.

\section{Mittlerer Atemwegsdruck}

Der Druck muss so hoch eingestellt werden, dass die Lunge des Patienten möglichst vollständig belüftet ist, ohne überbläht zu sein (Abb.3). Man nennt das eine High-Volume-Strategie [6].

Cave: Das Oszillieren in eine teilweise atelektatische Lunge ist zu vermeiden, da dies mit vermehrten Lungenschäden durch Scherkrafteinwirkungen auf das Epithel und auch Hirnblutungen in Zusammenhang gebracht wurde. Der Mechanismus für Letzteres ist unklar. 
Da sich die Compliance der Lunge im Krankheitsverlauf ändern kann, muss die Wahl des mittleren Atemwegsdrucks regelmäßig überprüft werden.

Merke: Es ist zu beachten, dass die Druckverhältnisse im Blutkreislauf und auch im Schädel mit der Einstellung des mittleren Atemwegsdrucks zusammenhängen.

Je höher der mittlere Atemwegsdruck ist, umso niedriger ist das Herzzeitvolumen und umso höher ist der intrakranielle Druck [7,8]. Dieser Zusammenhang wird jedoch modifiziert durch die pulmonale Compliance. Hatte in Tierversuchen die Lunge bei Surfactantmangel eine schlechte Compliance, wurde das Herzzeitvolumen durch den mittleren Atemwegsdruck deutlich weniger beeinträchtigt als bei einer gesunden Lunge [8].

In einer klinischen Studie konnte darüber hinaus nachgewiesen werden, dass das Lungenvolumen und der mittlere Atemwegsdruck in einem direkten Zusammenhang miteinander stehen [9]. Es kommt also sehr darauf an, den mittleren Atemwegsdruck individuell korrekt auf die mechanischen Eigenschaften der Patientenlunge einzustellen. Die Einstellung kann derzeit in der Routine nur anhand von Röntgenbildern des Thorax überprüft werden. Geeignet wären auch pulmonologische Messverfahren, wie die Messung der funktionellen Residualkapazität, die aber leider in der klinischen Routine für kleine Frühgeborene bis heute nicht zur Verfügung stehen.

Merke: Die richtige Einstellung des mittleren Atemwegsdrucks ist entscheidend für den Erfolg der Beatmung und sollte immer wieder hinterfragt und angepasst werden.

Das Flussdiagramm zeigt einen Algorithmus zur Einstellung des mittleren Atemwegsdrucks (Abb.3).

\section{Oszillationsamplitude}

Die Oszillationsamplitude ist der wesentliche Faktor bei der Eliminierung von Kohlendioxid. Je höher die Oszillationsamplitude, desto mehr $\mathrm{CO}_{2}$ wird aus der Lunge entfernt und umso niedriger wird der $\mathrm{PaCO}_{2}$ (arterieller Partialdruck des Kohlendioxids) des Patienten sein [10].

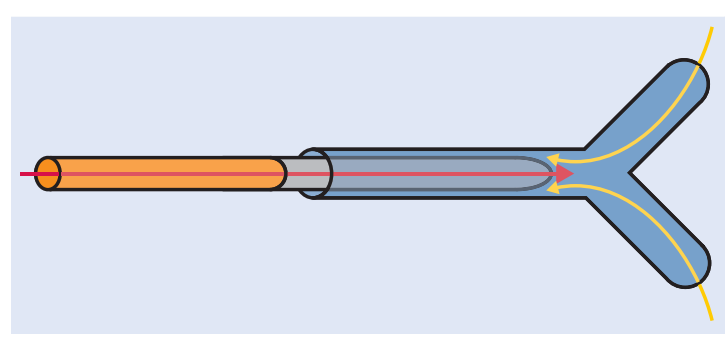

Abb. 4 Darstellung des Tubusendes in der Trachea und der Gasflüsse bei In- und Exspiration.

\section{Oszillationsfrequenz}

Üblicherweise werden Frequenzen zwischen 10 und 15 Hertz eingesetzt, da die Lungenimpedanz zwischen 10 und 30 Hertz ein Minimum aufweist [11] und die Geräte mit steigender Frequenz ineffektiver werden [12]. Deshalb steigt der $\mathrm{PaCO}_{2}$ nach Erhöhen der Frequenz, während ein Senken der Oszillationsfrequenz die $\mathrm{CO}_{2}$-Auswaschung verbessert.

\section{Inspirations- zu Exspirationsverhältnis}

Der Atemweg eines intubierten Patienten weist am Ende des Endotrachealtubus einen deutlichen Kalibersprung auf. Der Endotrachealtubus ist enger als der sich distal davon befindende nicht intubierte Teil der Trachea (Abb.4). Durch diesen Kalibersprung findet sich bei der Inspiration ein geringerer Strömungswiderstand als bei der Exspiration [13].

Als Konsequenz baut sich bei einer Hochfrequenzbeatmung mit symmetrischer Oszillation (d.h. die Inspiration ist gleich lang wie die Exspiration) intrapulmonal ein höherer Druck auf als am Y-Stück des Beatmungsgeräts. Diese Situation bezeichnet man als Air trapping, da die Luft in der Exspirationsphase weniger gut entweichen kann als sie bei der Inspirationsphase in die Lunge gelangt ist $[14,15]$. Demgegenüber ist eine Oszillation mit halb so langer Inspiration wie Exspiration (d.h. ein I: E-Verhältnis von 30\%) mit einem intrapulmonal niedrigeren Druck als am Y-Stück assoziiert.

Ist das I: E-Verhältnis frei einstellbar, scheint mir ein I:E-Verhältnis von $1: 1,5$, d.h. $40 \%$ der Periodendauer entfallen auf die Inspiration, ein günstiger Kompromiss zu sein.

Merke: Bei gleich langer In- und Exspirationsphase ist der intrapulmonale Druck unter HFOV etwas höher als der am Gerät eingestellte mittlere Atemwegsdruck. Um das zu vermeiden, sollte die Inspirationsphase etwas kürzer als die Exspirationsphase sein. 


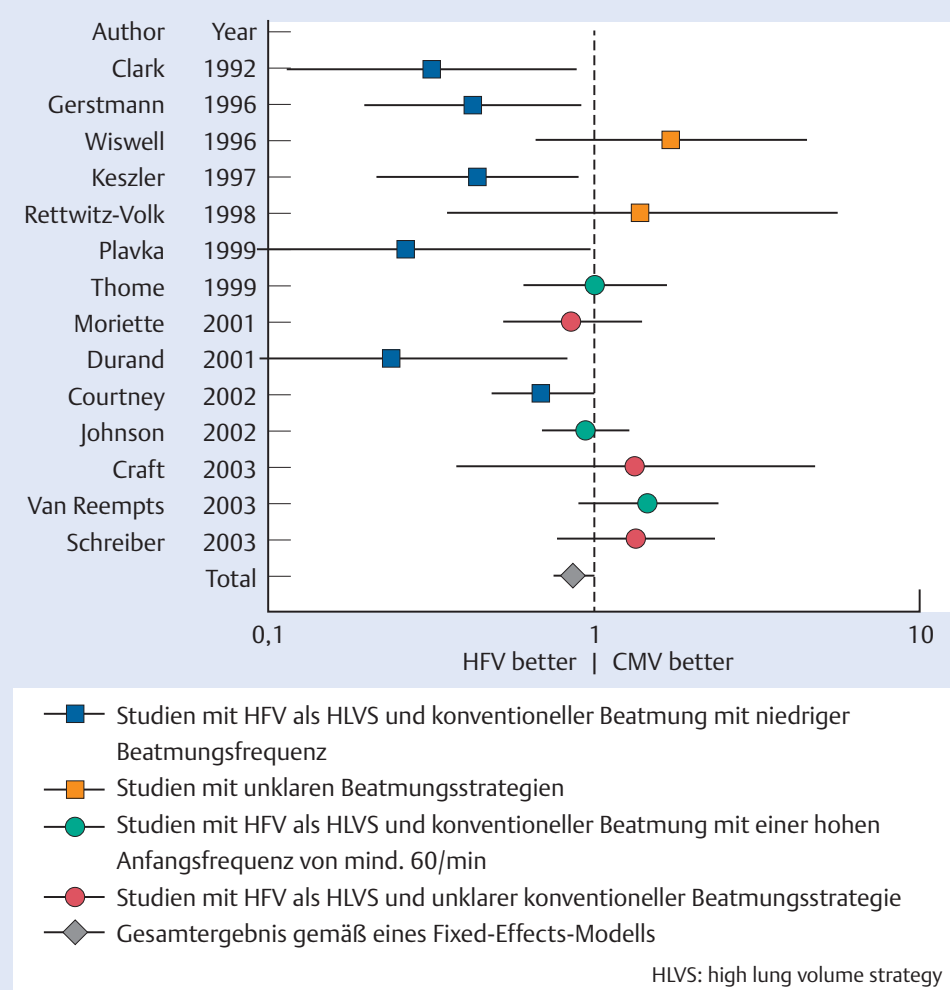

Abb.5 Metaanalyse der 14 Studien, die Hochfrequenzbeatmung mit konventioneller Beatmung verglichen und die Tod oder BPD in einem postmenstruellen Alter von 36 Wochen als primäres Zielkriterium hatten [16].

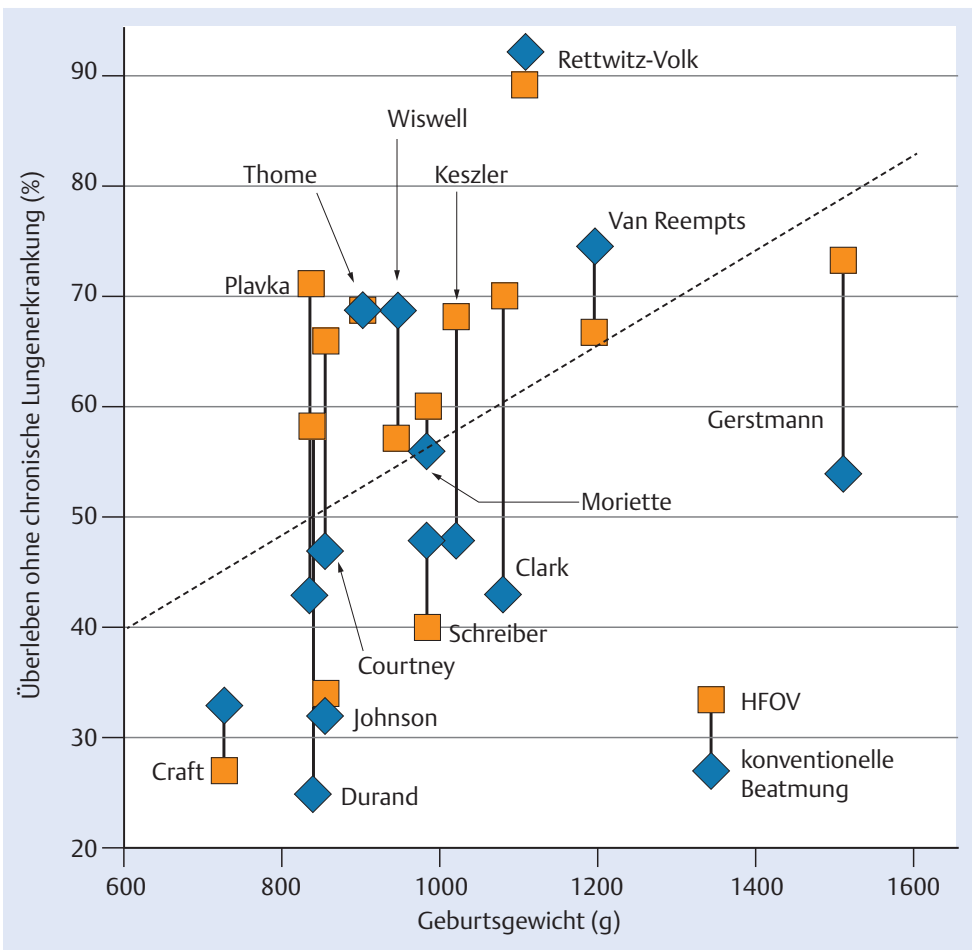

Abb. 6 Darstellung der Überlebensrate ohne chronische Lungenerkrankung in einem postmenstruellen Alter von 36 Wochen in allen Studien, die dieses Zielkriterium gemessen haben. Die gestrichelte Linie ist die Regressionslinie über alle Ergebnisse [16].

\section{Behandlungsergebnisse bei Frühgeborenen mit Atemnotsyndrom}

Die Wertigkeit von Hochfrequenzoszillationsbeatmung im Vergleich zu konventioneller Beatmung wurde in 19 randomisierten Studien an Frühgeborenen untersucht. Abb. 5 zeigt eine Metaanalyse aller 14 Studien, die als primäres Studienziel die Vermeidung von Tod oder bronchopulmonaler Dysplasie (BPD) mit 36 Wochen postmenstruell hatten. Studien mit verschiedenen Beatmungsstrategien wurden unterschiedlich markiert, je nachdem, ob die Beatmungsstrategie in den jeweiligen Studienarmen lungenschonend optimiert war [16]. Insgesamt gesehen ergab sich kein signifikanter Vorteil für die Hochfrequenzbeatmung. Ein gleiches Ergebnis hatte auch eine auf individuellen Patientendaten basierende Metaanalyse [17].

Man erkennt eine große Gruppe von Studien, in denen die Hochfrequenzbeatmung durch Anstreben eines optimalen Lungenvolumens (High-Volume-Strategie) mutmaßlich optimal durchgeführt wurde, nicht jedoch die konventionelle Beatmung hinsichtlich eines möglichst niedrigen Tidalvolumens durch möglichst hohe Beatmungsfrequenz. In diesen Studien hat die Hochfrequenzbeatmung deutlich bessere Ergebnisse erzielt als die konventionelle Beatmung.

Daneben gibt es eine Gruppe von 3 Studien, bei denen nicht nur die Hochfrequenzbeatmung wie beschrieben optimiert wurde, sondern auch die konventionelle Beatmung. In diesen Studien war die Hochfrequenzbeatmung der konventionellen Beatmung nicht überlegen.

Eine weitere Gruppe von Studien mit unklaren Beatmungsstrategien kann diesbezüglich nicht bewertet werden.

In Abb. 6 ist das Überleben ohne chronische Lungenerkrankung in Abhängigkeit vom mittleren Geburtsgewicht in den jeweiligen Armen der zitierten Studien aufgetragen. Die Linie zeigt die Regressionsgerade durch alle Ergebnisse und stellt somit das durchschnittliche Therapieergebnis dar. Man erkennt an dieser Grafik, dass Hochfrequenzbeatmung nur dann bessere Ergebnisse als die konventionelle Beatmung erzielt hat, wenn die Ergebnisse der konventionellen Beatmung unterdurchschnittlich waren.

Insgesamt kann aus diesen Ergebnissen geschlossen werden, dass eine Optimierung der konventionellen Beatmung Richtung Beatmungsfrequenzen von 60/min 
und höher und Minimierung des Tidalvolumens gleich gute Ergebnisse zu erzielen vermag wie die Hochfrequenzbeatmung.

Merke: Es kommt nicht auf die Wahl des Verfahrens an, sondern auf die optimale Durchführung.

Dies gilt zumindest für den Zustand der Kinder im postmenstruellen Alter von 36 Wochen.

Möglicherweise hat die Hochfrequenzoszillationsbeatmung jedoch Vorteile für Kinder mit besonders schwerer Lungenerkrankung. So hat z. B. eine italienische Arbeitsgruppe in einer neueren Studie nur Kinder randomisiert, die keine antenatale Glukokortikoidprophylaxe erhalten hatten. Sie fanden eine deutlich schnellere Entwöhnung von der Beatmung bei Kindern mit Hochfrequenzbeatmung [18].

Auch könnte die Lungenentwicklung der Kinder langfristig noch von der zuvor durchgeführten Hochfrequenzoszillationsbeatmung profitieren. Eine Nachuntersuchung von Teilnehmern der UKOS-Studie im Alter von 11 - 14 Jahren ergab eine signifikant bessere Lungenfunktion bei den Jugendlichen, die als Frühgeborene Hochfrequenzbeatmung erhalten hatten (Abb.7) [19], obwohl die während der stationären Therapie festgestellten Ergebnisse, gemessen an der Rate der bronchopulmonalen Dysplasie im postmenstruellen Alter von 36 Wochen, in beiden Gruppen fast exakt gleich gewesen waren. Auch waren die kognitiven Leistungen der mit HFOV beatmeten Kinder tendenziell besser und in keinem Aspekt schlechter als bei den konventionell beatmeten Kindern. Diese Ergebnisse deuten auch darauf hin, dass die Feststellung einer bronchopulmonalen Dysplasie im postmenstruellen Alter von 36 Wochen nur ein recht grobes Instrument zur Messung der postnatalen Lungengesundheit ist. Offenbar ist es möglich, mit genauer Lungenfunktionsmessung auch nach vielen Jahren noch Unterschiede herauszuarbeiten, die zuvor durch bloße Anwendung der klinischen BPD-Definition nicht nachweisbar waren.

Zusammenfassend sind bei Frühgeborenen die kurzfristigen Therapieergebnisse mit Hochfrequenzoszillationsbeatmung gleich gut wie mit einer optimierten konventionellen Beatmung. Trotz der beschriebenen, überwiegend unentschieden ausgegangenen Studien und Metaanalysen könnten mit Hochfrequenzoszillation beatmete Frühgeborene langfristig Vorteile im Sinne einer besseren Lungenfunktion haben. Auch deuten die italienischen Ergebnisse auf mögliche Vor-

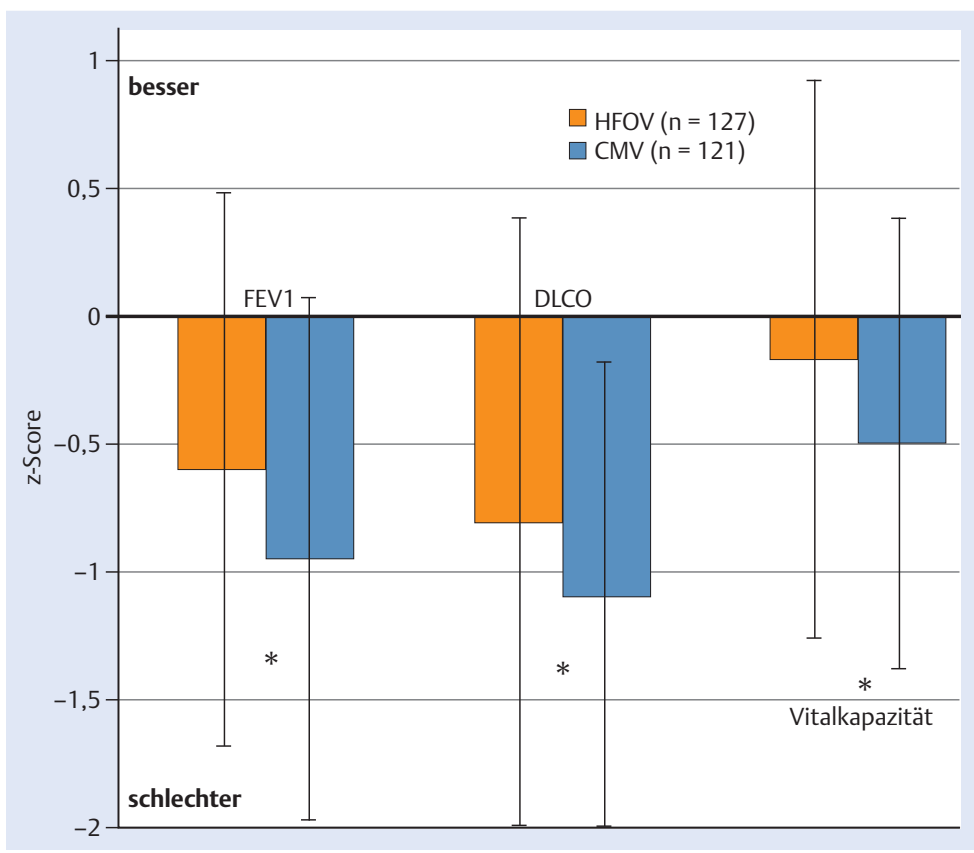

Abb. 7 Z-Scores verschiedener Lungenfunktionsparameter von ehemals frühgeborenen Jugendlichen, die HFOV oder CMV erhalten hatten [19]. (DLCO: Diffusionskapazität der Lunge für Kohlenmonoxid; FEV1: maximales Exspirationsvolumen in der ersten Sekunde [Tiffeneau-Test]; $\left.{ }^{*} \mathrm{p}<0,05\right)$.

teile der Hochfrequenzoszillation bei besonders schwer erkrankten Frühgeborenen hin.

Merke: Die Ergebnisse bei unkomplizierten Frühgeborenen sind mit HFOV nicht besser als mit einer optimierten konventionellen Beatmung. Bei

schwerem Lungenversagen und für die langfristige Lungenentwicklung könnte HFOV Vorteile haben.

Leider kann die Frage, welche Kinder am ehesten von Hochfrequenzbeatmung profitieren, aufgrund der vorliegenden Daten nicht beantwortet werden. Kandidaten sind aber alle Kinder, die früh eine erhebliche pulmonale Dysfunktion aufweisen.

Merke: Aufgrund der aktuellen Ergebnisse scheint es angebracht, wieder über einen verstärkten Einsatz der Hochfrequenzoszillation nachzudenken.

\section{Behandlungsergebnisse bei reifen Neugeborenen}

Nur 2 kleine randomisierte Studien haben die Ergebnisse der Hochfrequenzbeatmung bei reifen Neugeborenen untersucht. In einer Studie wurden zur extrakorporalen Membranoxygenierung (ECMO) verlegte Patienten randomisiert und mit Hochfrequenzoszil- 
lation oder konventioneller Beatmung behandelt, mit dem Ergebnis, dass weniger mit HFOV behandelte Kinder ECMO benötigten [20].

In einer weiteren randomisierten Cross-over-Studie wurde festgestellt, dass Hochfrequenzbeatmung die Lungenfunktion etwa so günstig beeinflusst, wie konventionelle Beatmung in Kombination mit inhalativem Stickstoffmonoxid. Für die Kombination beider Modalitäten, d.h. Hochfrequenzbeatmung mit Stickstoffmonoxid, wurde ein zusätzlicher synergistischer Effekt nachgewiesen [21]. Dies gilt möglicherweise nicht für Kinder mit Zwerchfellhernie. Deren Mortalität war in einer noch nicht publizierten Studie des europäischen ECMO-Netzwerks mit Hochfrequenzbeatmung höher als mit konventioneller Beatmung.

Ähnlich sieht es für Hochfrequenzbeatmung bei pädiatrischen und adulten Patienten mit Acute Respiratory Distress Syndrome (ARDS) aus. Für das pädiatrische ARDS gibt es eine relativ alte randomisierte Studie [22] und eine sehr große und neuere retrospektive Studie [23]. Bei ersterer waren die Ergebnisse bei HFOV zwar etwas besser, aber in der konventionell beatmeten Gruppe wurde vermutlich keine lungenprotektive Beatmung durchgeführt. In der zweiten Studie schnitt HFOV deutlich schlechter ab als konventionelle Beatmung mit längerer Beatmungsdauer und längerem Krankenhausaufenthalt.

In der Erwachsenenmedizin gab es 4 Studien; nur in der ältesten war HFOV der CMV überlegen, aber auch hier war die CMV nicht lungenprotektiv [24]. Weitere 2 z.T. sehr große Studien fanden keine Vorteile mehr $[25,26]$, und in einer vierten Studie war HFOV sogar klar schlechter [27]. Somit konnte auch für diese Patientengruppen kein Überlebensvorteil nachgewiesen werden.

Cave: Studien ergaben keine Vorteile für HFOV bei Kindern und Erwachsenen.

\section{Zusammenfassung}

Die Hochfrequenzoszillationsbeatmung ist einer optimierten konventionellen Beatmung hinsichtlich der kurzfristigen Behandlungsergebnisse nicht überlegen, könnte aber bei Frühgeborenen langfristig mit einer besseren Lungenfunktion assoziiert sein.

\section{Beatmung beim pulmonalen interstitiellen Emphysem}

Das pulmonale interstitielle Emphysem (PIE) stellt i.d.R. eine Komplikation der Beatmung dar, wenngleich es gelegentlich auch bei Continuous Positive Airway Pressure (CPAP) oder ganz selten auch bei einem spontan atmenden Kind beobachtet werden kann.

Wie der Name schon sagt, handelt es sich um Luftansammlungen im Interstitium, die sich bei Surfactantmangel und Flüssigkeitsüberschuss und dadurch schlecht dehnbare Alveolen und überdehnte terminale Bronchiolen bilden können. Da die Verbindung dieser pathologischen Hohlräume mit den Atemwegen nur sehr dünn ist und sie nicht von einem Kapillarsystem umsponnen werden, nehmen diese Luftansammlungen nicht am Gasaustausch teil. Sie stellen Raumforderungen dar, die das funktionierende Lungengewebe verdrängen und die Gasaustauschfläche reduzieren, sodass es i.d. R. zu einem deutlichen Anstieg des $\mathrm{FiO}_{2}-$ Bedarfs kommt.

Merke: Der deutliche Anstieg des $\mathrm{FiO}_{2}$-Bedarfs ist oft auch ein frühes klinisches Zeichen für die Entwicklung eines interstitiellen Emphysems.

Im weiteren Verlauf kann das interstitielle Emphysem immer mehr zunehmen und die Oxygenierung des Patienten immer weiter erschweren. Die Reaktion des klinischen Teams besteht dann i.d. R. in einem Anheben des Beatmungsdrucks, um das Kind weiterhin am Leben halten zu können, was jedoch die Entwicklung des Emphysems beschleunigt.

Cave: Eine adäquate Oxygenierung kann schwierig oder unmöglich werden, und als katastrophale Komplikation droht eine Luftembolie, wenn die Luft auch noch in das Gefäßsystem eindringt.

Das Emphysem kann jedoch auch in einen Zustand chronischer, schwerer pulmonaler Dysfunktion münden, der einer BPD sehr ähnlich ist, oder in eine BPD übergehen. Bei einem Fallbericht konnte die Persistenz des Emphysems über den errechneten Geburtstermin hinaus nachgewiesen werden, auch per Computertomografie, und später dann per Lungenhistologie [28].

Merke: Es handelt sich um eine Komplikation, die die Gesundheit der kleinen Patienten massiv und nachhaltig schädigen kann. 


\section{Risikofaktoren}

Die Risikofaktoren für die Entwicklung eines pulmonalen interstitiellen Emphysems sind:

- Frühgeburtlichkeit

- Surfactantmangel

- Behandlung mit Atemwegsüberdruck, insbesondere intensive maschinelle Beatmung

- Einsatz von Hochfrequenzoszillationsbeatmung

- Einsatz von langen Inspirationszeiten

\section{Diagnose}

Die Diagnose erfolgt i.d.R. durch ein Röntgenbild. Das Röntgenbild ist gekennzeichnet durch relativ harte Kontraste zwischen dem komprimierten Gewebe und den luftgefüllten pathologischen Hohlräumen. Diese werden in runde Blasen vom Typ 1 oder längliche Blasen vom Typ 2 [29] unterteilt. Nur das Vorhandensein von Blasen vom Typ 2 sichert die Diagnose (Abb.8).

\section{Prävention}

Präventiv wirken:

- pränatale Lungenreifeinduktion

- möglichst frühzeitige Surfactantgabe

- Verwendung einer lungenschonenden Beatmung mit niedrigen Spitzendrücken und kurzen Inspirationszeiten

Merke: Unter Hochfrequenzoszillationsbeatmung tritt das pulmonale interstitielle Emphysem häufiger auf, deshalb sollte diese Beatmungsform Frühgeborenen mit höhergradigem Atemnotsyndrom vorbehalten bleiben.

\section{Behandlung}

Das pulmonale interstitielle Emphysem ist potenziell reversibel, und die Reversibilität ist umso mehr gegeben, je früher eine gezielte Behandlung eingeleitet wird. Zur Behandlung sollten die physikalischen Gesetzmäßigkeiten so ausgenutzt werden, dass möglichst wenig Luftnachschub in die großen Blasen und die interstitiellen Spalten stattfindet, sodass die Resorption der dort vorhandenen Luft letztlich überwiegt und sich die überblähten Gewebsspalten wieder verschließen können.

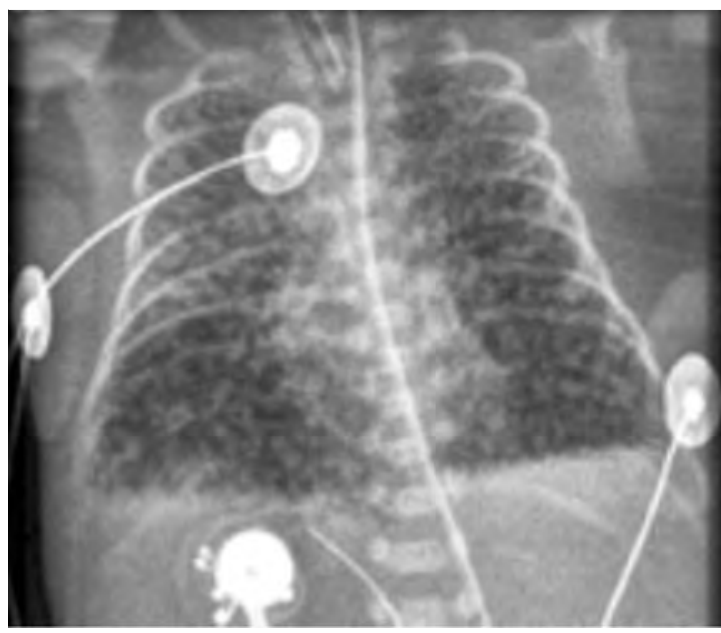

Abb. 8 Röntgenbild eines Frühgeborenen mit pulmonalem interstitiellen Emphysem. Deutlich erkennbar sind die länglichen Blasen, die harten Kontraste zwischen den Blasen und dem komprimierten Lungengewebe und die Überblähung der gesamten Lunge.

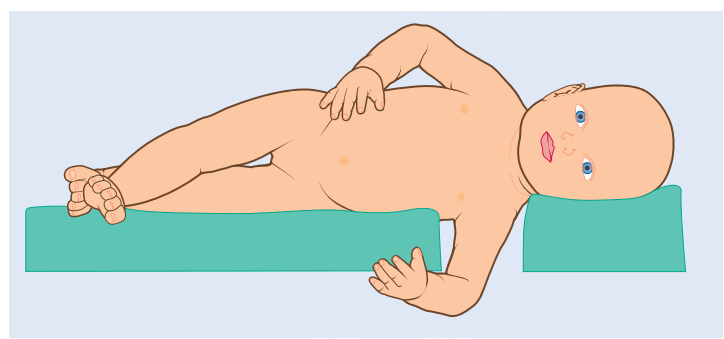

Abb. 9 Lagerung bei einseitigem pulmonalen interstitiellen Emphysem zur einseitigen Kompression des Thorax (die Schulter hängt frei).

Von Bedeutung ist hier zunächst die Reduktion des Tidalvolumens. Ist das pulmonale interstitielle Emphysem nur einseitig ausgebildet, kann das Tidalvolumen auf der betroffenen Seite durch Lagerung des Kindes auf die entsprechende Thoraxseite (Abb.9) oder durch Anbringen eines Tape-Verbands verringert werden, während der Gasaustausch vorwiegend über die andere Seite bewerkstelligt wird. Das sind auch die einzigen Behandlungsmöglichkeiten beim spontan atmenden Kind.

Merke: Ob es sinnvoll ist, ein unter Spontanatmung noch suffizient atmendes Kind zur Behandlung eines pulmonalen interstitiellen Emphysems zu intubieren, ist nicht geklärt.

Auch die einseitige Intubation wurde erfolgreich angewendet [30].

Cave: Große Vorsicht ist bei den Beatmungseinstellungen unter Lagerungstherapie vonnöten, um nicht auf der Gegenseite ein Emphysem zu induzieren. Es sollten hohe Beatmungsfrequenzen und kleine Tidalvolumina bevorzugt werden.

Die speziellen Beatmungseinstellungen umfassen als wesentlichen Faktor das Verkürzen der Inspirationszeit auf Werte zwischen 0,1 und 0,2 Sekunden [31]. Auf- 
Abb. 10 Überdehnte Alveolen und interstitielle Luftansammlungen haben längere Zeitkonstanten als normale Alveolen, weil ihre Compliance aufgrund des höheren Radius größer ist (La Place'sches Gesetz). Die Zeitkonstante $(\mathrm{T}=\mathrm{R} \times \mathrm{C})$ steigt mit steigender Resistance $(\mathrm{R})$ oder Compliance (C). Eine kurze Inspirationszeit ermöglicht deshalb eine selektive Reduktion des Tidalvolumens in den überdehnten Bereichen.

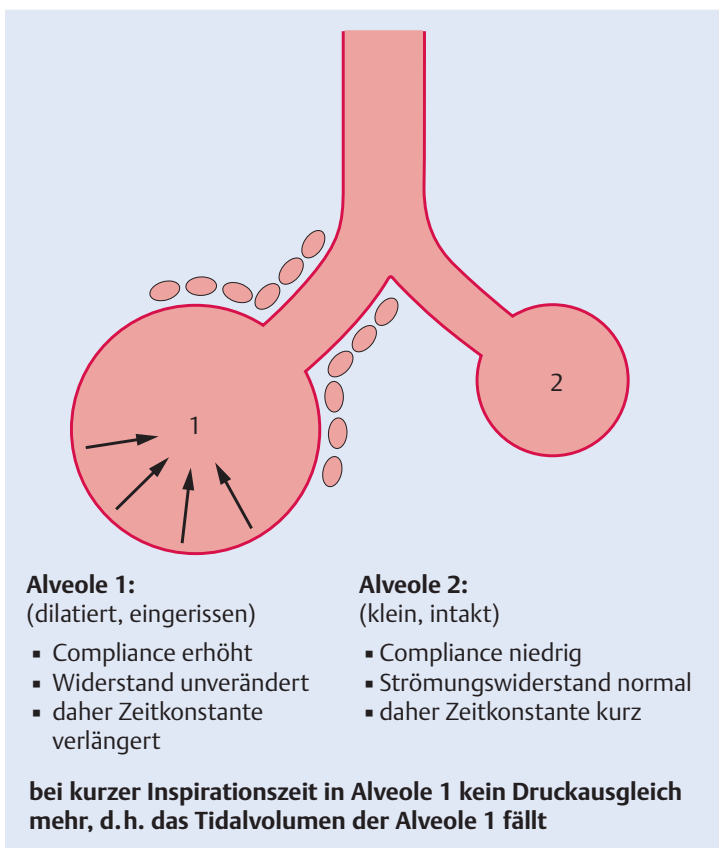

grund des hohen Strömungswiderstands durch die engen interstitiellen Spalten und ihrer dennoch leichten Dehnbarkeit weisen die Luftansammlungen des interstitiellen Emphysems sehr lange Zeitkonstanten auf $(\tau=R \times C)[1]$.

Sehr kurze Inspirationszeiten lassen keinen vollständigen Gasaustausch dieser Bereiche mehr zu und verringern somit selektiv das Tidalvolumen in den großen überblähten Alveolen und auch die Luftmenge, die in das interstitielle Emphysem fließt, während der Gasaustausch in den deutlich „schnelleren“ normalen Alveolen weniger beeinträchtigt wird. Dann kann man innerhalb von weniger als 24 Stunden oftmals einen erheblichen Rückgang der emphysematösen Veränderungen oder ein vollständiges Verschwinden beobachten (Abb. 10).

Merke: Surfactant darf erst nach Rückgang der
emphysematösen Veränderungen gegeben wer-
den, da dieses sonst die pathologischen Luftan-
sammlungen stabilisiert.

\section{Anmerkung des Autors}

Ich würde eine Surfactantgabe durchführen, bevor die Inspirationszeit wieder normalisiert wird. Eine Reduktion des endexspiratorischen Druckes (PEEP) ist nach meiner Erfahrung zur Behandlung eines pulmonalen interstitiellen Emphysems nicht notwendig.
Die Hochfrequenzoszillation mit niedrigem mittleren Atemwegsdruck wird immer wieder für die Behandlung des pulmonalen interstitiellen Emphysems empfohlen. Nach meinen Erfahrungen ist die hier beschriebene Methode mit kurzen Inspirationszeiten und Lagerung jedoch wesentlich erfolgreicher, sodass der Hochfrequenzoszillation hier keine Empfehlung ausgesprochen wird.

Merke: Die Behandlung eines pulmonalen interstitiellen Emphysems besteht in sehr kurzen Inspirationszeiten und Reduzieren des regionalen Tidalvolumens durch Lagerung oder Tapeverband.

\section{Zusammenfassung}

Die Hochfrequenzbeatmung ist eine Beatmungsform mit geringen alveolären Druck- und Volumenschwankungen. Die Einstellung des mittleren Atemwegsdrucks muss genau auf den Patienten abgestimmt und regelmäßig überprüft und ggf. korrigiert werden. In großen Studien konnte keine Reduktion der BPD bei Frühgeborenen unter HFOV gegenüber einer lungenprotektiven konventionellen Beatmung nachgewiesen werden. Nachuntersuchungen von ehemaligen Studienteilnehmern im Jugendalter ergaben jedoch Hinweise, dass HFOV längerfristig nachweisbare Vorteile haben könnte. Welche Frühgeborenen von HFOV profitieren, ist derzeit nicht geklärt. Für reife Neugeborene prekärer Oxygenierung konnten kurzfristige Vorteile im Zusammenhang mit HFOV nachgewiesen werden. Bei Kindern und Erwachsenen scheinen die Nachteile bei HFOV zu überwiegen.

Das pulmonale interstitielle Emphysem ist eine schwerwiegende Komplikation, bei der sich raumfordernde, aber nicht zum Gasaustausch beitragende Luftansammlungen im Interstitium der Lunge bilden. Die Rückbildung gelingt am ehesten durch eine konventionelle Beatmung mit extrem kurzer Inspirationszeit und - bei einseitiger Ausprägung - durch Reduktion des Tidalvolumens auf der betroffenen Seite mittels Lagerung oder Tape-Verband.

Interessenkonflikt: Kein Interessenkonflikt angegeben. 


\section{Über den Autor}

\section{Ulrich H. Thome}

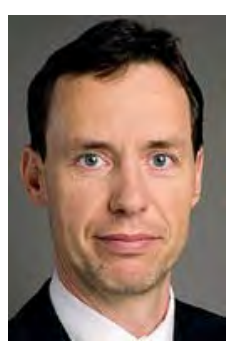

Jahrgang 1964, Univ.-Prof. Dr. med. Nach Medizinstudium und Promotion in Düsseldorf Ausbildung als Kinderarzt und pädiatrischer Intensivmediziner an der Universitätskinderklinik Ulm. Ab 1998 für 3 Jahre an der Universität von Alabama in Birmingham (USA) zur Forschung und gleichzeitig Ausbildung zum Neonatologen. Nach Rückkehr Oberarzt und Habilitation an der Universität Ulm. Seit 2008 Leiter der selbständigen Abteilung für Neonatologie am Universitätsklinikum Leipzig. Weiterbildungsermächtigung für Neonatologie und Pädiatrische Intensivmedizin.

\section{Korrespondenzadresse}

Prof. Dr. med. Ulrich H. Thome

Facharzt für Kinder- und Jugendmedizin/Neonatologie/ Pädiatrische Intensivmedizin

Abteilung Neonatologie der Universitätsklinik für

Kinder- und Jugendmedizin

Zentrum für Frauen- und Kindermedizin

Liebigstr. 20a, Haus 6

04103 Leipzig

ulrich.thome@medizin.uni-leipzig.de

\section{Literatur}

1 Thome UH. Mechanische Beatmung in der Neonatologie. Neonatologie Scan 2015; 4: 335-350

2 Lunkenheimer PP, Lunkenheimer A, Laval P et al. Experimental studies with high frequency oscillation. Int Anesthesiol Clin 1983; 21: $51-62$

3 Bohn D. High frequency oscillation. $\mathrm{Br}$ J Anaesth 1989; 63: 23

4 Pillow Jj. High-frequency oscillatory ventilation: mechanisms of gas exchange and lung mechanics. Crit Care Med 2005; 33 : $135-141$

5 Hummler HD, Thome U, Schulze A. Neue Beatmungsstrategien in der Neonatologie. Monatsschr Kinderheilkd 2002; 150: $669-682$

6 McCulloch PR, Forkert PG, Froese AB. Lung volume maintenance prevents lung injury during high frequency oscillatory ventilation in surfactant-deficient rabbits. Am Rev Respir Dis 1988; 137: 1185 - 1192

7 Walker AM, Brodecky VA, de Preu ND et al. High-frequency oscillatory ventilation compared with conventional mechanical ventilation in newborn lambs: effects of increasing airway pressure on intracranial pressures. Pediatr Pulmonol 1992; 12: $11-16$
8 Traverse JH, Korvenranta H, Adams EM et al. Impairment of hemodynamics with increasing mean airway pressure during high-frequency oscillatory ventilation. Pediatr Res 1988; 23 : $628-631$

9 Thome U, Töpfer A, Schaller P et al. Effect of mean airway pressure on lung volume during high-frequency oscillatory ventilation of preterm infants. Am J Respir Crit Care Med 1998; 157: $1213-1218$

10 Watson JW, Jackson AC. $\mathrm{CO}_{2}$ elimination as a function of frequency and tidal volume in rabbits during HFO. J Appl Physiol Respir 1984: 354 - 359

11 Dorkin HL, Stark AR, Werthammer JW et al. Respiratory system impedance from 4 to $40 \mathrm{~Hz}$ in paralyzed intubated infants with respiratory disease. J Clin Invest 1983; 72: 903 - 910

12 Fredberg JJ, Glass GM, Boynton BR et al. Factors influencing mechanical performance of neonatal high-frequency ventilators. J Appl Physiol 1987; 62: 2485 - 2490

13 Bush EH, Spahn DR, Niederer PF et al. Flow separation, an important mechanism in the formation of mean pulmonary pressure during high-frequency oscillation. J Biomech Eng 1989; 111: 17-23

14 Thome U, Pohlandt F. Effect of the TI/TE ratio on mean intratracheal pressure in high-frequency oscillatory ventilation. J Appl Physiol 1998; 84: 1520-1527

15 Pillow J], Neil H, Wilkinson $\mathrm{MH}$ et al. Effect of I/E ratio on mean alveolar pressure during high-frequency oscillatory ventilation. J Appl Physiol 1999; 87: 407-414

16 Thome UH, Carlo WA, Pohlandt F. Ventilation strategies and outcome in randomised trials of high frequency ventilation. Arch Dis Child Fetal Neonatal 2005; 90: F466-F473

17 Cools F, Askie LM, Offringa $M$ et al. Elective high-frequency oscillatory versus conventional ventilation in preterm infants: a systematic review and meta-analysis of individual patients' data. Lancet 2010; 375: 2082 - 2091

18 Salvo V, Zimmermann LJ, Gavilanes AW et al. First intention high-frequency oscillatory and conventional mechanical ventilation in premature infants without antenatal glucocorticoid prophylaxis. Pediatr Crit Care Med J Soc Crit Care Med World Fed Pediatr Intensive Crit Care Soc 2012; 13: 72 - 79

19 Zivanovic S, Peacock J, Cazar-Paris M et al. Late outcomes of a randomized trial of high-frequency oscillation in neonates. N Engl J Med 2014; 370: 1121 - 1130

20 Carter JM, Gerstmann DR, Clark RH et al. High-frequency oscillatory ventilation and extracorporeal membrane oxygenation for the treatment of acute neonatal respiratory failure. Pediatrics 1990; 85: 159- 164

21 Kinsella JP, Abman SH. Inhaled nitric oxide and high frequency oscillatory ventilation in persistent pulmonary hypertension of the newborn. Eur J Pediatr 1998; 157: 28 - 30

22 Arnold JH, Hanson JH, Toro-Figuero LO et al. Prospective, randomized comparison of high-frequency oscillatory ventilation and conventional mechanical ventilation in pediatric respiratory failure. Crit Care Med 1994; 22: 1530 - 1539

23 Gupta P, Green JW, Tang X et al. Comparison of high-frequency oscillatory ventilation and conventional mechanical ventilation in pediatric respiratory failure. JAMA Pediatr 2014; 168: $243-249$

24 Derdak S, Mehta S, Stewart TE et al. High-frequency oscillatory ventilation for acute respiratory distress syndrome in 
adults: a randomized, controlled trial. Am J Respir Crit Care Med 2002; 166: 801-808

25 Bollen CW, van Well GT], Sherry T et al. High frequency oscillatory ventilation compared with conventional mechanical ventilation in adult respiratory distress syndrome: a randomized controlled trial. Crit Care Lond Engl 2005; 9: R430 - R439

26 Young D, Lamb SE, Shah S et al. High-frequency oscillation for acute respiratory distress syndrome. N Engl J Med 2013; 368: 806-813

27 Ferguson ND, Cook DJ, Guyatt GH et al. High-frequency oscillation in early acute respiratory distress syndrome. $N$ Engl J Med 2013; 368: 795-805

28 Wilson JM, Mark EJ. Case 30-1997. N Engl J Med 1997; 337: $916-924$
29 Swischuk LE. Bubbles in hyaline membrane disease. Differentiation of three types. Radiology 1977; 122: 417-426

30 Jakob A, Bender C, Henschen $\mathrm{M}$ et al. Selective unilateral lung ventilation in preterm infants with acquired bullous emphysema: a series of nine cases. Pediatr Pulmonol 2013; 48: $14-19$

31 Gortner L, Pohlandt F, Bartmann P. Behandlung des unilateralen raumfordernden pulmonalen interstitiellen Emphysems mittels Lagerungsmassnahmen und höherfrequenter Beatmung. (Treatment of unilateral space- occupying pulmonary interstitial emphysema with positioning measures and highfrequency ventilation). Monatsschr Kinderheilkd 1988; 136: $432-435$ 


\section{CME-Fragen}

\section{CME.thieme.de}

\section{CME-Teilnahme}

- Viel Erfolg bei lhrer CME-Teilnahme unter http://cme.thieme.de

- Diese Fortbildungseinheit ist 12 Monate online für eine CME-Teilnahme verfügbar.

- Sollten Sie Fragen zur Online-Teilnahme haben, unter

http://cme.thieme.de/hilfe finden Sie eine ausführliche Anleitung.

\section{1}

Was gilt für die Hochfrequenzoszillationsbeatmung?
A Die Amplitude wird unverändert in die peripheren Atemwege übertragen.

B Die typischerweise verwendete Atemfrequenz liegt über 20 Hertz.

C Die Oxygenierung wird durch den mittleren Atemwegsdruck bestimmt.

D Die $\mathrm{CO}_{2}$-Elimination steigt mit steigender Frequenz.

E Sie sollte mit einem mittleren Atemwegsdruck von wenigstens 5 mbar über der konventionellen Beatmung durchgeführt werden.

\section{2}

Die Hochfrequenzoszillationsbeatmung
A verringert erwiesenermaßen die Häufigkeit der BPD.
B ist das Standardverfahren für die Beatmung Frühgeborener.
C verhindert das Auftreten eines interstitiellen Emphysems.
D ist vorteilhaft bei reifen Neugeborenen mit schwerem Lungenversagen.
E erfordert mehr Sedierung als konventionelle Beatmung.

\section{3}

Zum Einstellen der Hochfrequenzbeatmung muss folgender Parameter nicht vorgewählt werden:
A inspiratorischer Spitzendruck
B mittlerer Atemwegsdruck
C Inspirations- zu Exspirationsverhältnis
D Oszillationsamplitude
E Oszillationsfrequenz

4

Bei gleich langer In- und Exspirationszeit ist der mittlere Druck in der Lunge bei der Hochfrequenzbeatmung
A niedriger als im Beatmungsschlauchsystem.
B gleich hoch oder höher als im Beatmungsschlauchsystem.
C abhängig vom Gerät.
D unabhängig vom mitteren Druck im Beatmungsschlauchsystem.
E ohne vorhersagbare Tendenz.

\section{5}

Die Steuerung der Oxygenierung erfolgt bei HFOV durch
A die Oszillationsamplitude.
B die Oszillationsfrequenz.
C den mittleren Atemwegsdruck.
D das I:E-Verhältnis.
E den Bias-Flow.

6

Die Steuerung der Ventilation $\left(\mathrm{CO}_{2}\right.$ Auswaschung) erfolgt bei Hochfrequenzbeatmung hauptsächlich durch
A die Oszillationsamplitude.
B die Oszillationsfrequenz.
C den mittleren Atemwegsdruck.
D das I:E-Verhältnis.
E den Bias-Flow. 


\section{CME-Fragen}

Spezielle Beatmungssituationen in der Neonatologie

\section{7}

Welche Aussage ist richtig? Metaanalysen zeigen,
A dass HFOV unabhängig von der Strategie der CMV unterlegen ist.
B dass es von der gewählten Strategie in beiden Vergleichsgruppen abhängt, welche Beatmung überlegen ist.
C dass HFOV unabhängig von der Strategie der CMV überlegen ist.
D dass HFOV besonders für sehr kleine Kinder nützlich ist.
E dass die Ergebnisse der verschiedenen Studien gut reproduzierbar waren.

\section{8}

In einer Nachuntersuchung im Alter von 11 - 14 Jahren hatten Kinder, die mit HFOV behandelt worden waren,

9

Welche Aussage zum pulmonalen interstitiellen Emphysem ist falsch?
A einen Gehörschaden.
B eine schlechtere Lungenfunktion als die Vergleichsgruppe.
C schlechtere schulische Leistungen.
D vermehrt Konzentrationsstörungen.
E eine bessere Lungenfunktion als die Vergleichsgruppe.

A Es ist mit der Entwicklung einer BPD assoziiert.

B Es kann in das Säuglingsalter hinein fortbestehen.

C Es kann auch unter Spontanatmung auftreten.

D Es stellt nur eine alveoläre Überblähung dar.

E Es kann mit stark verschlechterter Sauerstoffaufnahme einhergehen.

10

Beim akuten Auftreten eines pulmonalen interstitiellen Emphysems ist welche Maßnahme sinnvoll?
A Beatmung mit niedriger Frequenz
B sofortige Surfactantgabe
C Erhöhen des PEEP
D Beatmung mit sehr kurzen Inspirationszeiten
E Beatmung mit HFOV 